\title{
Reorganising for COVID-19 Pandemic: A Review of Structural Modifications by the Department of Internal Medicine in Low to Middle-income Countries
}

\author{
Shakeel ur Rehman ${ }^{1}$, Muhammad Arslan Ahmed ${ }^{1}$, Ghufran Adnan ${ }^{1}$ and Maria Ali Khan ${ }^{2}$ \\ ${ }^{1}$ Department of Medicine, The Aga Khan University Hospital, Karachi, Pakistan \\ ${ }^{2}$ Department of Biostatistics and Epidemiology, The Aga Khan University Hospital, Karachi, Pakistan
}

\begin{abstract}
Scientists classified the severe acute respiratory syndrome coronavirus 2 (SARS-CoV-2) as a novel coronavirus on January 7 , 2020. The World Health Organization (WHO) acknowledged the SARS-CoV-2 outburst a public health emergency of international concern on January 30, 2020. Since its origin, this virus has disrupted the best healthcare systems, economies, and strained financial resources; and for underdeveloped countries' healthcare systems, the virus has become a crisis. To tackle the potential hazards from this virus, our Department restructured the services that not only helped us to cope with the pandemic, but also provided an example to copy for other contemporary institutes. This article aims to describe the measures and structural changes undertaken by the Internal Medicine Department, Aga Khan University Hospital (AKUH), Karachi, Pakistan.
\end{abstract}

\section{Key Words: COVID-19, Internal medicine, Structural modifications, Pandemic.}

How to cite this article: Rehman SU, Ahmed MA, Adnan G, Khan MA. Reorganising for COVID-19 Pandemic: A Review of Structural Modifications by the Department of Internal Medicine in Low to Middle-income Countries. J Coll Physicians Surg Pak 2020; 30(JCPSPCR):CR176-CR177.

The importance of appropriate education and training for hospital staff, including physicians, nurses, and other healthcare personnel, is essential for preventing the in-patientspread of Coronavirus Disease 2019 (COVID-19). ${ }^{1}$ The rate of intra-hospital infections and transmission has been reported to be up to 3.8\% among healthcare workers. Individual training sessions have been arranged regarding donning and doffing of personal protective equipment (PPE) as per CDC guidelines. Literature has proven that lack of knowledge of proper donning and doffing techniques canlead to self-contamination. ${ }^{2}$

Mandatory qualitative fitness testing for all healthcare workers was designed. For those, who were unable to pass Fit Test while wearing N95 masks, respirator masks were arranged; and if still, the test was negative, they were assigned to non-COVID areas. The reason behind undertaking this process was to correct the process from the previous SARS outbreak during which infected healthcare workers had not been Not Fit-tested. ${ }^{3}$ It was noted that due to beards, many healthcare workers failed the Fit Test with the $\mathrm{N} 95$ masks, although they were able to get through the test with respirator masks.

Correspondence to: Dr. Ghufran Adnan, Department of Medicine, Aga Khan University Hospital, Stadium Road, Karachi, Pakistan

E-mail:drghufranadnan@gmail.com

Received: September 01, 2020; Revised: November 02, 2020; Accepted: November 03, 2020

DOI: https://doi.org/10.29271/jcpsp.2020.JCPSPCR.CR1 76

This finding indicated that respiratormasks are essential for hospi- tals with bearded healthcare workers.

For employee health screening, a 24/7 helpline and screening app were established. For all suspected infected healthcare workers, diagnostic testing with nasopharyngeal swab polymerase chain reaction (PCR) was done; and those who tested positive, were managed as per standard protocols. All exposed employees were immediately quarantined, and regularly enquired about symptoms. To reduce the spread of COVID-19 among healthcare workers, early identification and isolation of suspected staff is essential. $^{4}$

This new challenge warranted a workforce transformation and some radical changes that were made to the duty roster. Due to exposure, only a few of the residents and interns quarantined. Doctors, including residents, interns, and medical officers, were split into two groups, with each group working on alternate weeks. This rotation was planned as a way to minimise COVID-19 exposure and keep an adequate workforce reserved for handling the expected COVID-related patient surge. As the patient admission rate in internal medicine drastically increased, workers were taken from other subspecialties. This pandemic has also revealed that every hospital must provide a helping hand and facilitate excellent communications among all departments to reduce the burden on the healthcare staff.

In order to educate healthcare workers regarding the management and care of COVID-19 patients, tutorials and webinars were arranged on Microsoft teams and ZOOM. ${ }^{5}$ This format was used to minimise any physical contact withouthampering educational needs.

As this software was new for many healthcare workers, they 
required some time to familiarise themselves with these formats. This process has alerted us to the need for continuing online sessionsto learn new technology even after the pandemic ends.

Outpatient services were disrupted during the initial phase of the pandemic. To minimise any delay in delivering healthcare to patients, the teleclinic facility was launched immediately. Shifting of clinics to telemedicine was difficult because of a lack of software availability among patients and healthcare staff. This difficulty raises the point that low- to middle-income countries require funding for accessibility and awareness of telemedicine services to provide timely care to patients. ${ }^{6}$

Due to an increased number of COVID-19 patients entering the hospital, a need for an urgent negative pressure unit to accommodate all critical, suspected, and definite cases of COVID-19 and to minimise emergency department (ED) personnel exposure was felt. The acute care unit was changed into negative pressure remote unit on an urgent basis in mid-March, 2020. All suspected patients who came to the ED were first admitted to this unit. We suggest preventing overwhelming an emergency area in a pandemic by providing a negative pressure unit near an ED to which they can quickly transfer the patients.

Due to an increased number of COVID-19 patients, there was a need for an isolated COVID-19 facility. The building was modified into a negative pressure isolation facility to accommodate moderately to severely ill COVID-19 patients. Separate donning and doffing areas were provided and regular audits were done. The deficiencies were discussed by audit teams in regular meetings with hospital directors, and any issues were promptly fixed and corrected. Even in pandemic situations, audits help to rectify mistakes and improve patient care.

All patients were admitted to internal medicine for COVID-19 testing before being referred to their specific sub-specialty. These patients were not admitted to their specialties due to the risk of exposure. Swabs were sent for COVID-19 testing, and those patients who reported negative were returned to concerned specialties; while those who were positive, were admitted under the internal medicine service and assigned a specialist who visited them regularly. As it is challenging to differentiate asymptomatic COVID-19 patients, we recommend performing a COVID test before admitting any patient during a pandemic.
Failure to cope with the COVID-19 surge can result in the collapse of any healthcare facility; and because of interruptions to public health systems, may lead to enormous economic losses. It is irrelevant that interventions may ultimately control this global COVID-19 outbreak, but sharing experiences and measures to contain COVID-19 in hospitals is of the utmost importance.

This detailed account proposes to guide contemporary institutes in coping with this pandemic and successfully steering through it.

\section{CONFLICT OF INTEREST:}

Authors declared no conflict of interest.

\section{AUTHORS' CONTRIBUTION:}

SR, MAA: Wrote the main manuscript.

GA, MAK: Reviewed the article and made final changes.

\section{REFERENCES}

1. Wong J, Goh QY, Tan Z, Lie SA, Tay YC, Ng SY, et al. Preparing for a COVID-19 pandemic: A review of operating room outbreak response measures in a large tertiary hospital in Singapore. Can J Anesth 2020; 67(6):732-45. doi: 10.1007/s12630-020-01620-9.

2. Casanova LM, Teal LJ, Sickbert-Bennett EE, Anderson DJ, Sexton DJ, Rutala WA, et al. CDC Prevention Epicenters Program. Assessment of self-contamination during removal of personal protective equipment for Ebola patient care. Infec Control Hosp Epidemiol 2016; 37(10):1156-61. doi: 10.1017/ice.2016.169.

3. Centers for Disease Control and Prevention (CDC. Cluster of severe acute respiratory syndrome cases among protected health-care workers--Toronto, Canada, April 2003. MMWR. Morbidity and mortality weekly report 2003; 52(19):433.

4. Zhao Y, Cui C, Zhang K, Liu J, Xu J, Nisenbaum E, et al. COVID19: A systematic approach to early identification and healthcare worker protection. Frontiers in Public Health 2020; 8:205. doi: 10.3389/fpubh.2020.00205.

5. Barbosa TJ, Barbosa MJ. Zoom: An innovative solution for the live-online virtual classroom. HETS Online J 2019; 9:2.

6. Ahmed A, Ahmed M. The telemedicine landscape in Pakistan-why are we falling behind? J Pak Med Assoc 2018; 68(12):1820-2. 